\title{
Assessing interactions, predicting function, and increasing degradation potential of a PAH-degrading bacterial consortium by effect of an inoculant strain
}

\author{
Marianela Macchi ${ }^{1} \cdot$ Sabrina Festa ${ }^{1}$ - Nelson E. Vega-Vela ${ }^{2,3} \cdot$ Irma S. Morelli $^{1,4} \cdot$ Bibiana M. Coppotelli $^{1}$ (D)
}

Received: 22 February 2019 / Accepted: 14 June 2019

(C) Springer-Verlag GmbH Germany, part of Springer Nature 2019

\begin{abstract}
A natural phenanthrene-degrading consortium CON was inoculated with an exogenous strain Sphingobium sp. (ex Sp. paucimobilis) 20006FA yielding the consortium called I-CON, in order to study ecological interactions into the bacterial community. DGGE and proteomic profiles and analyses by HTS (High-Throughput Sequencing) technologies demonstrated inoculant establishment and changes on CON composition. Inoculation increased degradation efficiency in I-CON and prevented intermediate HNA accumulation. This could be explained not only by the inoculation, but also by enrichment in Achromobacter genus at expense of a decrease in Klebsiella genus. After inoculation, cooperation between Sphingobium and Achromobacter genera were improved, thereby, some competition could have been generated, and as a consequence, species in minor proportion (cheaters), as Inquilinus sp. and Luteibacter sp., were not detected. Sequences of Sphingobium (corresponding to the inoculated strain) did not vary. PICRUSt predicted a network with bacterial phylotypes connected with enzymes, showing functional redundancy in the phenanthrene pathway, with exception of the first enzymes biphenyl-2,3-diol 1,2-dioxygenase and protocatechuate 4,5-dioxygenase that were only encoded in Sphingobium sp. This is the first report where a natural consortium that has been characterized by HTS technologies is inoculated with an exogenous strain in order to study competitiveness and interactions.
\end{abstract}

Keywords Inoculation/invasion - Bacterial consortia $\cdot$ Degradation potential $\cdot$ Invasion $\cdot$ Predicted functions $\cdot$ PICRUSt · Proteome

\section{Introduction}

The focus of the ecological systems biology studies interactions within and between species to elucidate community-

Responsible editor: Robert Duran

Electronic supplementary material The online version of this article (https://doi.org/10.1007/s11356-019-05760-4) contains supplementary material, which is available to authorized users.

Bibiana M. Coppotelli

bibicoppotelli@gmail.com

1 Centro de Investigación y Desarrollo en Fermentaciones Industriales, CINDEFI (UNLP; CCT-La Plata, CONICET), Street 50 №227, 1900 La Plata, Argentina

2 Pontificia Universidad Javeriana, Bogotá, Colombia

3 Universidad de Bogotá Jorge Tadeo Lozano, Bogotá, Colombia

4 Comisión de Investigaciones Científicas de la Provincia de Buenos Aires, La Plata, Argentina level properties such as diversity and stability. To do so, theory and experiments in simple laboratory systems are integrated, in which general principles can be tested systematically (Friedman and Gore 2017). Environmental pollution is one of the most relevant challenges of our time, in terms of its potential adverse effect on biodiversity of natural ecosystems and human health (Megharaj et al. 2011). Biological degradation is the primary dissipation mechanism for most organic pollutants in soil environment (Cerniglia and Yang 1984; Cerniglia 1993). Different bacteria dominate specific processes of biodegrading hydrocarbons or are able to complete the degradation by consuming intermediate metabolites generated by other members of the community.

It has been proposed that the inoculation of strains or microbial consortia can be beneficial to improve the degradation rates of organic pollutants in contaminated sites (De Lorenzo et al. 2013). The design of bioaugmentation strategies requires knowledge of the potential of the inoculated microorganisms to improve the degradation efficiency and its influence in the native microbial community. Bacterial consortia have 
advantages over isolated strains to achieve an efficient bioremediation process (Wanapaisan et al. 2018) since they create a network with different levels of interaction that show a better adaptation, survival, and permanence (Zafra et al. 2017), giving them strength to face environmental changes (Brenner et al. 2008). In addition, bacterial consortia are good models to study the interactions that can occur between bacterial populations during a bioremediation process (Desai et al. 2010). Interactions among members may include physical contact, chemical signaling, and metabolic exchange.

Improved biocatalysts/biodegraders can be designed for expanding the substrate ranges of existing pathways, for improving the substrate flux through pathways to avoid the accumulation of inhibitory intermediates or for increasing the genetic stability of catabolic activities and the bioavailability of hydrophobic pollutants. One strategy for designing enhanced catalysts for bioremediation is improving the biodegradative performance of a consortium through the addition of a "specialist" organism (Timmis and Pieper 1999). Little is known about how individual bacterial taxa of different bacteria function in the degrading process (Wang et al. 2016) and about how microbes cooperate mutually (Tao et al. 2017). Nowadays, bioinformatic approaches called "predictive metagenomics" has been developed, and based on the presumption that phylogeny (obtained from High-Throughput Sequencing technologies) and function are linked, predicting the functional composition of a metagenome (Su et al. 2018). Relative distributions of functional genes - KEGG orthologs (KOs) - can be inferred from OTUs relative abundance data by using bioinformatic tools such as PICRUSt (Langille et al. 2013).

We aimed to design a microbial consortium to analyze a simpler biological system to study the competitiveness of a microorganism introduced in an established bacterial community. For that purpose, we inoculate a previously obtained natural consortium CON (Festa et al. 2013), with a foreign microorganism, found to be efficient in $\mathrm{PAH}$-degradation: Sphingomonas paucimobilis 20006FA strain (Coppotelli et al. 2008), yielding the consortium called I-CON.

The selected consortium, CON, was obtained from a PAH chronically contaminated soil. Its diversity, structure, and functionality were studied during phenanthrene degradation in liquid medium by classical and molecular methods (Festa et al. 2013, 2016, 2017). It was observed that the consortium failed to completely reduce phenanthrene supplied during 15 days of incubation and produced the accumulation of metabolites which might lead to a toxicity affecting cell growth (Yuan et al. 2000; Zhao et al. 2008).

Sphingomonas paucimobilis 20006FA was designated as a good candidate to be used as an inoculant in the remediation of soils contaminated with $\mathrm{PAH}$, due to its ecological properties and its ability to mineralize phenanthrene (Coppotelli et al. 2010). Recently, its genome and proteome were analyzed predicting putative gene-encoding enzymes for all the steps of phenanthrene degradation, which loci could also participate in the metabolism of other PAH (Macchi et al. 2018). Nevertheless, the accumulation of 1-hydroxy-2-naphthoic acid (HNA), the intermediate metabolite in phenanthrenedegradation pathway, was detected both in soil and in pure cultures when phenanthrene concentration exceeded $800 \mathrm{mg} / \mathrm{l}$ (Coppotelli et al. 2008, 2010; Macchi et al. 2018). For that reason, it was proposed that this strain could better work in bacterial consortia probably allowing cooperative metabolic activities. To achieve an efficient bioremediation process, it is important to find bacteria involved in the performance of a complete degradation pathway, so that potentially toxic metabolites do not accumulate (Kazunga and Aitken 2000). It was suggested that co-cultures of Sphingomonas strains with other bacterial genera, which allow cooperative metabolic activities, can reduce the concentration of toxic metabolites of PAH degradation (Yuan et al. 2000; Zhao et al. 2008).

\section{Materials and methods}

\section{Design and study of bacterial consortium I-CON}

A phenanthrene-degrading bacterial consortium $(\mathrm{CON})$ was obtained from a chronically PAH-contaminated soil, as detailed in previous investigations (Festa et al. 2013). It was conserved in $15 \%$ of glycerol at $-80^{\circ} \mathrm{C}$ and when required it was reactivated in liquid mineral medium (LMM) containing $5 \mathrm{~g} \mathrm{NaCl}, 1 \mathrm{~g} \mathrm{~K}_{2} \mathrm{HPO}_{4}, 1 \mathrm{~g}\left(\mathrm{NH}_{4}\right) \mathrm{H}_{2} \mathrm{PO}_{4}, 1 \mathrm{~g}\left(\mathrm{NH}_{2}\right)_{2} \mathrm{SO}_{4}$, $0.2 \mathrm{~g} \mathrm{MgSO}_{4}, 3 \mathrm{~g} \mathrm{KNO}_{3}$, and $1000 \mathrm{~cm}^{3}$ of de-ionized water at a pH of 7.2, supplemented with $200 \mathrm{mg} / \mathrm{l}$ of phenanthrene for 7 days. The relative frequency of phylotypes in $\mathrm{CON}$ revealed the presence of six bacterial genera belonging to the Proteobacteria phylum (Festa et al. 2016). These data were updated in 2017 according to the SILVA database (Quast et al. 2012) (http://www.arb-silva.de). At genus level, the following relative abundances were observed in decreasing order: Sphingobium sp. (88.1\%), Klebsiella sp. (6.6\%), Achromobacter sp. (2.8\%), Inquilinus sp. (1.3\%), Luteibacter sp. (0.7\%), and Pseudomonas sp. (0.5\%).

Sphingomonas paucimobilis 20006FA strain (GenBank under the accession number LYMJ00000000) was obtained from a bioremediation experiment on a soil microcosm artificially contaminated with phenanthrene and characterized by Coppotelli et al. (2008).

Recently 20006FA strain was taxonomically reclassified according to the species re-alocation within genus Sphingomonas and Sphingobium suggested by Zhao et al. (2017). In this sense, we performed a re-evaluation of the 16S rRNA gene sequence of S. paucimobilis 20006FA (GenBank: DQ400860.1) with respect to Sphingobium genus. The taxonomic reclassification performed by NCBI resulted in an update in the name of the genome organism WGS to Sphingobium sp. 20006FA. 
However, for reasons of consistency with the works previously published with this strain, we decided to continue using the term Sphingomonas "sensu latu" to refer to it. The broad denomination "sensu latu" is applied to name bacteria belonging to the genus Sphingomonas, which has four subgenera proposed by Takeuchi et al. (2001), called Sphingobium, Novosphingobium, Sphingopyxis, and Sphingomonas (sensu stricto).

Natural consortium CON was inoculated with Sphingobium sp. 20006FA strain, to generate I-CON. Cultures containing $1.10^{6} \mathrm{cfu} / \mathrm{ml}$ (counted on R2A) of both reactivated consortium $(\mathrm{CON})$ and 20006FA strain, were incubated in LMM (Vecchioli et al. 1990) supplemented with $200 \mathrm{mg} / \mathrm{l}$ of phenanthrene as sole carbon and energy sources for 15 days at $28^{\circ} \mathrm{C}$ and $150 \mathrm{rpm}$.

\section{PAH degradation}

Phenanthrene and HNA degradation in CON and I-CON were determined in triplicate in LMM with 200 and $50 \mathrm{mg} / \mathrm{l}$, respectively, as sole carbon and energy sources. Cultures were incubated at $28^{\circ} \mathrm{C}, 150 \mathrm{rpm}$ and monitored at 0-, 4-, 7-, and 15-day intervals. The total culture volume of each Erlenmeyer was extracted three times with ethyl acetate (1:5 ethyl acetate/ culture ratios) at each incubation time. A triplicate abiotic control sample was also analyzed. In order to determine the presence of HNA, a sequential extraction was carried out. After the third extraction with ethyl acetate, the remnant aqueous phase was acidified to $\mathrm{pH} 2.5$ with concentrated $\mathrm{HCl}$ and re-extracted with ethyl acetate in the same way (Coppotelli et al. 2010). The ethyl acetate extracts were analyzed by reversed-phase highpressure liquid chromatography (HPLC), using a Waters ${ }^{\circledR}$ chromatograph with a Symmetry Waters ${ }^{\circledR}$ C18 column $(15 \mathrm{~cm} \times 4.6 \mathrm{~mm}$ i.d.; bead size, $5 \mu \mathrm{m}$; pore size, $100 \AA)$ and a diode- array detector. A linear gradient of $15 \mathrm{mM}$ phosphoric acid in nanopure water solution and methanol (20:80 to 5:95, $\mathrm{vol} / \mathrm{vol}$ ) over $15 \mathrm{~min}$ and a flow rate of $1 \mathrm{ml} / \mathrm{min}$ was used (Coppotelli et al. 2010; Festa et al. 2013).

Statistical analyses of degradation data were performed by a parametric one-way ANOVA test, using the SigmaPlot/ SigmaStat software program (SPSS Inc., Chicago, Illinois, USA).

Same protocol was carried out when analyzing the degradation of anthracene, dibenzothiophene, fluorene, and pyrene in cultures of I-CON with $200 \mathrm{mg} / \mathrm{l}$ of PAH as sole carbon source.

\section{Analyses of bacterial diversity and proteomic profiles of CON and I-CON}

Bacterial diversity in CON and I-CON consortia was examined in terms of structure and composition, during phenanthrene degradation, through the determination of bacterial counts (heterotrophic cultivable bacteria and PAH-degrading bacteria), PCR-DGGE and 16SrRNA gene pyrosequencing. Proteomic profiles were studied through SDS-PAGE analyses.
Heterotrophic cultivable bacteria counts were quantified in duplicates by colony-forming unit (cfu) counting on R2A (Reasoner and Geldreich 1985) medium plates. Colonies were differentially counted along incubation period. PAH-degrading microbial population counts were determined in sterile polypropylene microplates according to Wrenn and Venosa (1996) using the most-probable-number (MPN) technique, as previously detailed (Coppotelli et al. 2010; Festa et al. 2013).

Denaturant gradient gel electrophoresis (DGGE) analyses were performed with extracted DNA from CON and I-CON at $0,4,7$, and 15 days of incubation period as reported by Festa et al. (2016). The set of primers used for PCR amplification were GC-341F (5'-CGCCCGCCGCGCCCCGCGCC CGGCCCGCCGCCCCCGCCCCCTCCTACGGGAGGCAG CAG-3') and 907R (5'-CCGTCAATTCCTTTGAGTTT-3') (Muyzer and Smalla 1998). DGGE were performed in a DGGE-2000 System (CBS Scientific Company). The purified PCR products were directly loaded in a $(6 \% \mathrm{w} / \mathrm{vol})$ polyacrylamide gel (acrylamide- $N, N^{\prime}$-methylenbisacrylamide, 37.5:1) containing a linear gradient of $45-70 \%$ denaturant (100\% denaturant corresponds to $7 \mathrm{M}$ urea and $40 \% \mathrm{vol} / \mathrm{vol}$ formamide). The electrophoretic run was executed at a temperature of $60^{\circ} \mathrm{C}$ at a constant voltage of $100 \mathrm{~V}$ for $16 \mathrm{~h}$.

Total protein extraction of 20006FA strain and CON and ICON cultures in LMM supplied with $200 \mathrm{mg} / \mathrm{l}$ of phenanthrene after 4 and 15 days of incubation, was performed according to previous investigations (Festa et al. 2017). SDS-PAGE (12\%) was conducted in a Mini PROTEAM Tetra Cell (Bio-Rad, Hercules, CA) at 100 V. SDS-PAGE gels were calibrated with a molecular mass marker PageRulerTM Prestained Protein Ladder (10-170 kDa) (Pierce Endogen). The separated protein bands were stained with Coomassie brilliant blue G-250 and gel images captured with Universal Hood II (Bio-Rad, Hercules, CA). All the studies were performed in triplicate.

Samples of DNA from CON and I-CON consortia corresponding to day four of incubation in phenanthrene cultures were used for PCR amplification using the 16S rRNA universal bacterial primers, 341Fbac (CCTACGGGAGGCAGCAG) (Muyzer et al. 1993) and 909R (CCCCGYCAATTCMTTTRAGT) (Tamaki et al. 2011) to amplify a 568-bp fragment of the $16 \mathrm{~S}$ rRNA gene flanking the V3 and V4 regions. Samples were sequenced utilizing Roche 454 FLX titanium instrument and reagents at the Molecular Research laboratory (MR DNA; Shallowater, TX) (http://www.mrdnalab.com/) (Festa et al. 2016). Raw sequence data are archived at the Genome Short Read Archive (SRA) at the National Center for Biotechnology Information (NCBI) (SUB4848058).

\section{Analyses of pyrosequencing data}

In order to estimate bacterial taxa abundances in the $16 \mathrm{~S}$ rRNA datasets of CON and I-CON and functional roles in terms of KOs, the amplified 16S rRNA gene fragments were analyzed 
using the Microbiome Helper workflow (Comeau et al. 2017): this includes the use of QIIME (Caporaso et al. 2010), FastQC (v0.11.5) (http://www.bioinformatics.babraham.ac.uk/projects/ fastqc/), and PEAR (v0.9.10) (Zhang et al. 2014) in the first steps in order to evaluate raw reads, identify ambiguous reads, and stitch them together. After chimeric screening and removal using VSEARCH (v1.11.1) (Rognes et al. 2016) and UCHIME algorithm (Edgar et al. 2011) respectively, open-reference OTU picking was performed at $97 \%$ identity using SortMeRNA (Kopylova et al. 2012) and SUMACLUST (Mercier et al. 2013) and reads were clustered against the Greengenes database. Prior to the normalization of the final OTU table, low confidence OTUs were removed. A BIOM file was formatted to be used as an input for PICRUSt (Langille et al. 2013).

A gene copy number normalization and KEGG ortholog prediction were carried out with the BIOM file to obtain the contributions of various taxa to different KOs (Comeau et al. 2017).

Finally, to assess the diversity of consortium I-CON, a family of diversity indices developed by Mark Hill (Hill 1973), called Hill numbers, were used. Hill numbers $\left({ }^{\mathrm{q}} \mathrm{D}\right)$ are defined by a "q" order (Gotelli and Chao 2013) and this parameter determines the sensitivity of the measure to the relative abundances of the species (Chiu and Chao 2014), and the most used are: the species richness $\left({ }^{0} \mathrm{D}\right)$, the Shannon entropy exponential $\left({ }^{1} \mathrm{D}\right)$, and the inverse of the Simpson index $\left({ }^{2} \mathrm{D}\right)$.

\section{Network visualization}

The occurrence of genes in bacterial phylotypes involved in phenanthrene metabolism can be represented as a network. A bipartite network, bigraph or 2-mode network, $G=(V, E)$ is one in which the nodes can be partitioned into two disjoint sets, $V_{1}$ and $V_{2}$, such that $(u, v) \in E$ implies either $u \in V_{l}$ and $v$ $\in V_{2}$, or $v \in V_{l}$ and $u \in V_{2}$ (Pavlopoulos et al. 2011, 2018). Let $G=(V, E)$ comprise a set $V_{1}$ of metabolic genes predicted by PICRUSt, a set $V_{2}$ denote the bacterial phylotypes present in the bacterial consortium CON or I-CON, and a set $E$ represent the occurrence of a gene in a particular bacterial phylotype. Matrix $B$ represents the min-max normalized contribution values - calculated using PICRUSt - $|U| \times|V|$ biadjacency matrix of $G(V, E)$ where $B_{i k}$ denotes the min-max normalized contribution value of the edge connecting a bacterial phylotype $V_{1}$ and a metabolic gene (KO term) $V_{2}$.

These bipartite networks were constructed using Cytoscape 3.6.1 (Shannon et al. 2003). In order to generate them, a simple plain text file containing bacterial phylotypes (vertex set 1), KO terms (vertex set 2), and some node and edge attributes (e.g., min-max normalized contribution values, quartiles, and metabolic step), was used as an input file. In these networks, an edge connects a vertex from set 1 - bacterial phylotype - to a vertex from set 2 - KO term - if PICRUSt predicts the occurrence of a gene - KEGG ortholog — contributed by a specific bacterial phylotype to the consortium (e.g., CON or I-CON). The size of nodes represented the degree of a node, which indicates the number of edges it has to other nodes, in our case, the number of genes involved in phenanthrene metabolism that a bacterial phylotype has. In addition, the normalized contribution values are represented by the stroke edge color (a gray-to-blue scale) and the width edge was set to its count gene contribution quartile (or 4-quantiles). We also calculated the biadjacency matrix from each of the bipartite networks for describing equivalences between the consortia. Biadjacency matrices and stack bar plots were created using ggplot2 (Wickham 2016) in the R environment (version 3.5.1, http://www.r-project.org).

\section{Results}

\section{Comparative phenanthrene biodegradation and HNA production by bacterial consortia CON and I-CON}

The kinetics of phenanthrene degradation in LMM supplemented with $200 \mathrm{mg} / 1$ of phenanthrene was studied in ICON and compared with CON (Festa et al. 2013) and 20006FA strain. Figure 1 allows to infer that, whereas after seven 7 days the percentage of degraded phenanthrene was similar in both bacterial consortia and close to $50 \%$, I-CON managed to eliminate $78 \%$ after 15 days of incubation, which resulted in significantly higher $(P<0.05)$ degradation efficiency than the percentage reached by CON (59\%). The 20006FA strain was able to degrade around $99 \%$ of phenanthrene after 15 days of incubation (Macchi et al. 2018).

Concentration of HNA was quantified during the incubation time in both bacterial consortia (Fig. 1). While in CON, an increase of the intermediary concentration was observed during the first 7 days of incubation, reaching a value around

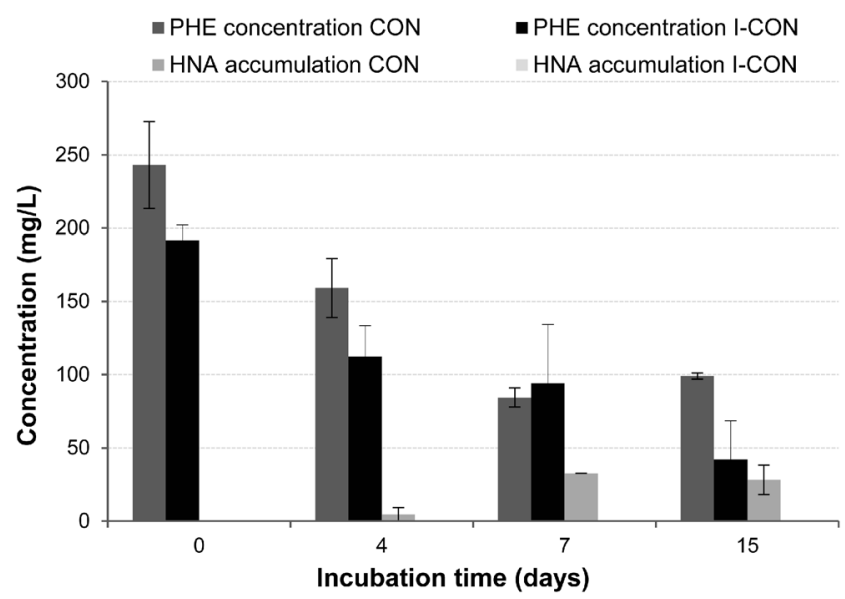

Fig. 1 Phenanthrene and 1-hydroxy-2-naphthoic acid (HNA) concentration in CON and I-CON cultures in LMM with $200 \mathrm{mg} / \mathrm{l}$ of phenanthrene as sole carbon and energy sources during 15 days of incubation. Results are means of triplicate independent experiments. Bars represent standard deviations 
$28 \pm 10 \mathrm{mg} / \mathrm{l}$ (which remained constant until the day 15 ), in ICON there was no evidence of intermediary accumulation. However, if we consider the stoichiometric values, where all the phenanthrene that is degraded generates HNA, in a mol-tomol equation $\left(\mathrm{C}_{14} \mathrm{H}_{10}+5 \mathrm{O}_{2} \rightleftharpoons \mathrm{C}_{11} \mathrm{H}_{8} \mathrm{O}_{3}+\mathrm{H}_{2} \mathrm{O}+3 \mathrm{CO}_{2}\right)$, part of the HNA that produced $\mathrm{CON}$ would be degraded at the same speed as that produced, leaving only $13.4 \%$ remaining on day 15 of incubation. In the case of I-CON, the total of HNA generated during the reaction could be used by the microbial community, without being accumulated.

When kinetics of HNA degradation was studied in cultures supplemented with exogenous HNA (50 mg/l) as sole carbon and energy sources, both consortia showed similar elimination percentages (around 84\%) after 15 days of incubation (Supplementary Fig. S1). Is worth to notice that in these cultures, the concentration of HNA used is four times lower than what would be generated when phenanthrene is degraded $(200 \mathrm{mg} / \mathrm{l})$.

The degradation profile of $\mathrm{CON}$ and I-CON were studied in LMM with different PAH (200 mg/l of anthracene, dibenzothiophene, fluorene, and pyrene) as sole carbon and energy sources and incubated for 15 days (Table 1). The PAH degrading profiles were differential, being broader that of ICON that showed greater degradation efficiency of dibenzothiophene $(38 \pm 8 \%)$ in relation to $\mathrm{CON}(24 \pm 2 \%)$ and anthracene degradation $(19 \pm 3 \%)$. No degradation was detected for fluorene and pyrene in any of the consortia. However, there was no difference in the degradative profile between I-CON and 20006FA strain.

\section{Effect of inoculation with Sphingobium sp. 20006FA on bacterial diversity and population dynamics and proteomic profiles of CON during phenanthrene degradation}

\section{Counts}

The effect produced by the inoculation on the dynamics of the cultivable populations of $\mathrm{CON}$ was first determined by

Table 1 Degradation percentage of different PAH in cultures of 20006FA strain, CON, and I-CON growing in LMM with $200 \mathrm{mg} / \mathrm{l}$ of $\mathrm{PAH}$ as sole carbon and energy source during 15 days incubation

\begin{tabular}{llll}
\hline & \multicolumn{2}{l}{ Degradation percentage (\%) } \\
\cline { 2 - 4 } PAH & $20006 \mathrm{FA}$ & CON & I-CON \\
\hline Anthracene & $13 \pm 11$ & - & $19 \pm 3$ \\
Dibenzothiophene & $76 \pm 3$ & $24 \pm 2$ & $38 \pm 8$ \\
Phenanthrene & 99 & 78 & 59 \\
Fluorene & - & - & - \\
Pyrene & - & - & - \\
\hline
\end{tabular}

Results are means \pm standard deviations of three biological replicates -: not detected analyzing changes in heterotrophic cultivable bacteria (HB) and PAH-degrading bacteria (PAH-DB) in both bacterial consortia during 15 days of incubation (Fig. S2).

In consortium $\mathrm{CON}$, the number of $\mathrm{HB}$ increased during the first days of incubation, showing its maximum value on day 4. From that day, a decay of two orders of magnitude was observed until the end of the assay (Fig. S2a black line). Inoculation with 20006FA strain caused a significant increase in HB counts that was maintained throughout the incubation time, and surpassed the values of CON with almost up to two orders of magnitude at the end of the assay (Fig. S2b black line). A differential bacterial plate counting was carried out in consortium I-CON considering color of colonies (Supplementary Fig. 2).

$\mathrm{PAH}$-degrading bacteria evolution in $\mathrm{CON}$ and I-CON was monitored throughout the treatment using the MPN method. CON presented a maximum count value on day 7 of incubation (Fig. S2a, dotted black lines) and then suffered a decrease towards day 15 , reaching lower values than the initial ones by four orders of magnitude.

In I-CON, the number of PAH-degrading bacteria remained constant throughout the assay (Fig. S2b, dotted black lines), exhibiting at the end of the incubation time, a PAH-degrading bacteria count five orders higher than CON.

\section{DGGE}

In order to compare the structure and the population dynamics of CON and I-CON consortia by molecular methods, a PCR was carried out with specific primers for the 16S rRNA gene, using the DNA of both bacterial consortia at different times during phenanthrene degradation. Then, from these samples of CON and I-CON, a DGGE was performed. As shown in supplementary material Fig. S5, the DGGE of 20006FA strain, CON and I-CON was analyzed to identify the bands corresponding to the consortium and to the inoculum (marked with a yellow arrow).

Although several bands were observed in the DGGE profile of 20006FA strain (what could be due to the multiple copies of 16S rRNA genes present in Proteobacteria (Větrovský and Baldrian 2013); the predominant band (marked with a yellow arrow: Fig. S5) was clearly observed in I-CON until the end of the assay (Fig. 2a), evidencing the establishment of the strain within the natural consortium CON (Fig. 2a).

When comparing CON and I-CON profiles at the four monitored times, it resulted in a concordance with bacterial counts (Fig. S2). Whereas a dynamic in the populations throughout the assay was observed in $\mathrm{CON}$, as evidenced by the three bands that appeared on day 15 (light blue arrows1, 2, and 3); in I-CON the structure of the community remained constant throughout the assay, no dynamic was observed (Fig. 2a), as in bacterial counts (Fig. S2). 


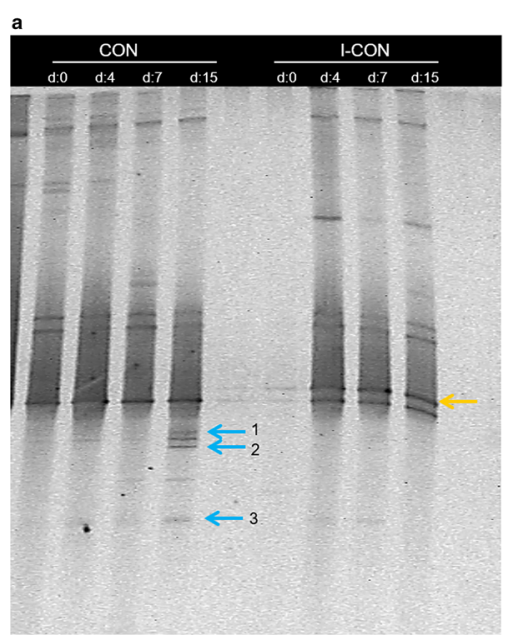

b

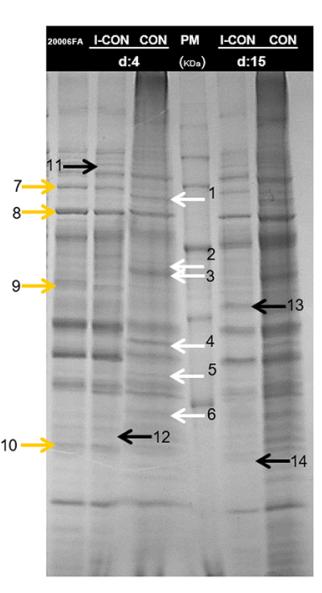

Fig. 2 Dynamics of bacterial populations during phenanthrene degradation. PCR-DGGE analyses of bacterial populations in CON and I-CON (a) and Polyacrylamide Gel Electrophoresis (12\% SDS-PAGE) analyses of 20006FA, CON, and I-CON cultures (b). Lane MW: molecular weight marker

\section{Proteomic profiles of both consortia}

Proteomic profiles of CON and I-CON were studied through comparative proteomic analyses at 4 and 15 days during phenanthrene degradation through SDS-PAGE gels (Fig. 2b).

The proteomic analyses showed that in I-CON, many of protein bands, that were present in $\mathrm{CON}$, were not visible as a result of the inoculation with 20006FA strain (marked with white arrows 1, 2, 3, 4, 5, and 6). In addition, the appearance of new protein bands in I-CON would correspond to the inoculated strain (yellow arrows 7, 8, 9, and 10). Furthermore, in I$\mathrm{CON}$, the protein banding pattern changed at the different incubation times during phenanthrene degradation (black arrows $11,12,13$, and 14).

\section{Pyrosequencing}

High throughput sequencing technique was applied to further analyze taxonomic composition of I-CON and to compare it with CON composition, previously analyzed by Festa et al. (2016). Pyrosequencing analyses of $16 \mathrm{~S}$ rRNA gene PCR fragments were performed from total DNA of I-CON obtained at day 4 of incubation time.

Pyrosequencing generated a total of 1501 filtered sequences that were grouped into 15 OTUs (operational taxonomic units) considering a minimum of $97 \%$ similarity. All representative sequences of each OTU were classified within Bacteria domain and Proteobacteria phylum (Alpha, Gamma and Beta, $84 \%, 3.8 \%$ and $12.2 \%$ of the reads respectively). Rarefaction curves of CON and I-CON (Supplementary Fig. S6) tended to approach the saturation plateau. This result was supported by the Good's index values, which reports the coverage of the analyses (Table 2). Values near 1.0 indicate that the obtained sequences for each library represent the majority of the bacterial sequences in the sample (Rungrassamee et al. 2013).

A decrease in species richness caused by inoculation was observed in I-CON, which is indicated by a lower value of Hill ${ }^{0} \mathrm{D}$ number (Table 2) in relation to $\mathrm{CON}$. The rarefaction curves confirmed this difference (Fig. S6) that was also observed in the DGGE patterns (Fig. 2a). Moreover, the diversity index (Hill ${ }^{1} \mathrm{D}$ number) in I-CON was lower than these of CON (Table 2), indicating a decrease in the diversity of indigenous bacterial consortium as a consequence of inoculation.

The taxonomic assignments are summarized in Fig. 3. I-CON showed a significant increase in the percentage of sequences belonging to Achromobacter sp. (11.5\%) in relation to $\mathrm{CON}(2.8 \%)$, at the expense of a significant decrease in the percentage of Klebsiella sp. sequences $(2.1 \%)$ in relation to $\mathrm{CON}(6.6 \%)$; and a slightly decreased in the sequences belonging Sphingobium sp. (genera corresponding to the inoculated strain), $84.4 \%$ in ICON and $88.1 \%$ in CON. Additionally, bacteria belonging to Luteibacter and Inquilinus genera were not detected in I-CON (Fig. 3).

\section{Assessing contribution of bacterial populations of CON and I-CON consortia to the cooperative metabolic pathway during phenanthrene degradation}

Functional genes encoding 29 enzymes in all bacterial OTUs found in CON and I-CON, involved in phenanthrene degradation pathway (Figs. 4a and 5), were predicted by PICRUSt using Greengenes database. A biadjacency matrix and a bipartite network showing the relationships between six bacterial phylotypes found in $\mathrm{CON}$ and I-CON with their enzymatic functions - KO terms - is presented in Fig. 4a-c. Note the most obvious change between the matrices, as well the networks, is the extinction of Luteibacter sp. and Inquilinus sp. phylotypes in consortium I-CON, and subtle changes as a consequence of it. In $(b-c)$, gray light dots represent bacterial

Table 2 Diversity parameters for CON and I-CON, obtained by analyses of pyrosequencing data using EstimateS program

\begin{tabular}{lcllllll}
\hline & $\begin{array}{l}\text { Number of } \\
\text { sequences }\end{array}$ & $\begin{array}{l}\text { Number } \\
\text { of OTUs }\end{array}$ & & \multicolumn{2}{l}{ Hill numbers } & & Good's index \\
\cline { 3 - 5 } & & & ${ }^{0} \mathrm{D}$ & ${ }^{1} \mathrm{D}$ & ${ }^{2} \mathrm{D}$ & \\
\hline $\mathrm{CON}$ & 996 & 21 & 22 & 1.51 & 2.24 & 0.99 \\
$\mathrm{I}-\mathrm{CON}$ & 1501 & 15 & 15 & 1.29 & 2.49 & 0.99 \\
\hline
\end{tabular}

${ }^{a}$ Values based on 905 random sequences per sample 


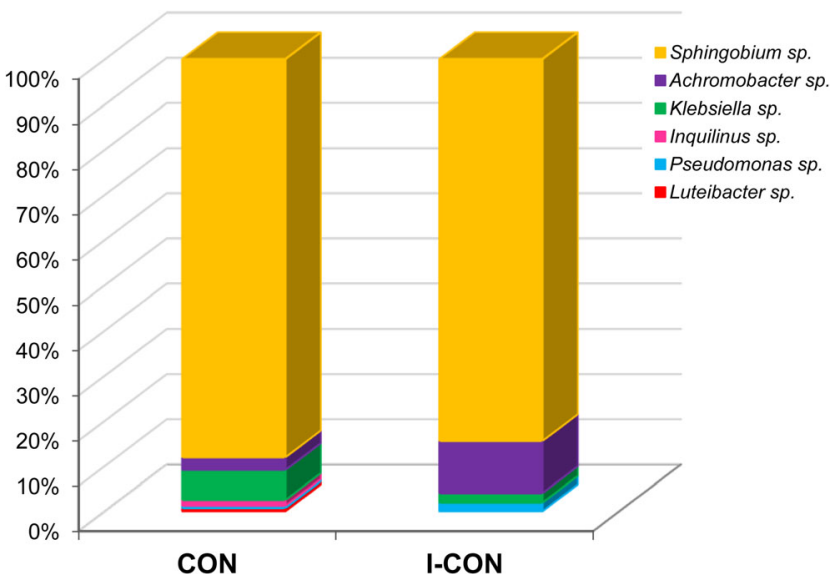

Fig. 3 Relative phylotype frequency at the genus level in CON and I$\mathrm{CON}$ as revealed by pyrosecuencing at day 4 of incubation during phenanthrene degradation. Affiliation was determined with a level of similarity of $97 \%$ using BLASTn against a curated Greengenes database

phylotypes without dramatic changes produced by strain inoculation after 4 days of incubation; bacterial phylotypes that significantly increased or significantly decreased after strain inoculation are represented with orange and aquamarine dots, respectively.

According to the prediction of PICRUSt, Sphingobium sp. was considered as the unique functional bacterial group able to perform two key reactions, catalyzed by enzymes biphenyl-2,3-diol 1,2-dioxygenase (K00462) and protocatechuate 4,5-dioxygenase (K04101), both responsible for the initial attack to phenanthrene. In addition, enzyme biphenyl 2,3-dioxygenase (K08689), necessary for the transformation of the intermediary phthalic acid (PA) is also codified in Sphingobium sp. (step 10 in Fig. 5). However, no significant changes were observed in the contribution of Sphingobium genus after 20006FA strain inoculation (Fig. 4a, c).

After inoculation, a negative impact in the contribution of Klebsiella genus and a positive impact in Achromobacter genus modified the contribution for several KO (Fig. 4a, c). While in consortium CON, Pseudomonas sp., Sphingobium sp., and Klebsiella sp. were the principal groups coding for 2,3-dihydro-2,3dihydroxybenzoate dehydrogenase (K00216) and dihydroxycyclohexadiene carboxylate dehydrogenase (K05783) enzymes, responsible for the second metabolic step of phenanthrene degradation pathway (Figs. 4a-c and 5); in I-CON, a significant increase in contribution of Achromobacter sp. for the K05783 was observed, this group becoming the one contributing most to the second step (Fig. 4a-c).

The four following steps, catalyzed by the enzymes maleylacetoacetate isomerase (K01800), 4carboxymuconolactone decarboxylase (K01607), 4-hydroxy 2-oxovalerate aldolase (K01666), and acetaldehyde dehydrogenase (K04073), involved in the process of degrading phenanthrene to 1-hydroxy-2-naphthoic acid (HNA), were predicted in all six bacterial phylotypes present in CON.

As a consequence of inoculation, the contribution of Klebsiella sp. to the enzymes acetaldehyde dehydrogenase (K04073) and 4-carboxymuconolactone decarboxylase (K01607), responsible for the fourth and fifth steps, significantly decreased. In addition, Luteibacter sp. and Inquilinus sp. populations, that were minority in consortium CON, extinguished in I-CON (Fig. 4c).

Phenanthrene degradation can be performed by orthoand meta-cleavage pathways (Fig. 5). In I-CON, the most significant change in enzyme contribution by genus predicted by PICRUSt occurred in the group of enzymes involved in the degradation of HNA by ortho-cleavage to phthalic acid. In this low region of the pathway, the contribution of Achromobacter genus also increased significantly for the enzyme aldehyde dehydrogenase (K00128), with a concomitant decreased in Klebsiella genus contribution.

In meta-cleavage pathway (from HNA to salicylic acid), Achromobacter sp. contribution increased for protocatechuate 3,4-dioxygenase (K00449, K00448) and homogentisate 1,2-dioxygenase (K00451), with concomitant decrease in Klebsiella sp. contribution for the same enzymes (Figs. 4a and 5).

Finally, in I-CON, the metabolic steps involved in the transformation of salicylic acid to TCA cycle were dominated by Achromobacter sp. who increased the contribution to the enzyme catechol 1,2-dioxygenase (K03381).

Considering the effect of inoculation regarding $\mathrm{KO}$ terms related to dioxygenase enzymes, in the step 3 of phenanthrene degradation pathway, the contribution rate of Achromobacter genus increased from 6.8 to $24.5 \%$ (more than 10\%) while the contribution of Klebsiella genus decreased from 23.7 to $5.6 \%$ (more than 10\%). Within the group of $\mathrm{KO}$ terms related to dehydrogenase enzymes, the contribution rate of Achromobacter genus increased from 3.3 to $15.1 \%$, while the contribution of Klebsiella genus decreased from 19.8 to $4.8 \%$ (Fig. 4a-c). No important changes were observed regarding the contribution of Sphingobium genus or other populations, for dioxygenase and dehydrogenase activities.

\section{Discussion}

The use of bioaugmentation strategies for the treatment of contaminated sites must be preceded by a series of laboratory tests and microcosms studies, in order to evaluate the capacity of the selected microorganisms to degrade the contaminant and to colonize the soil. It is therefore necessary to adopt an 
a

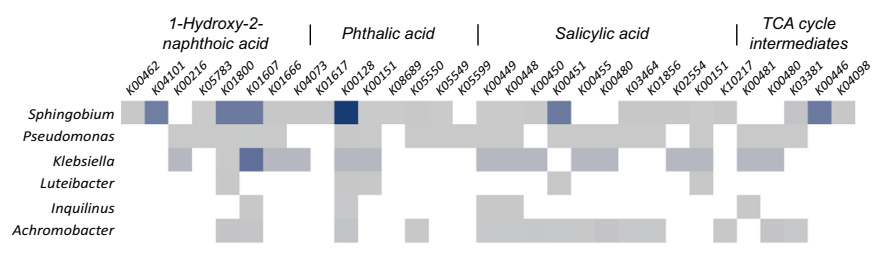

b

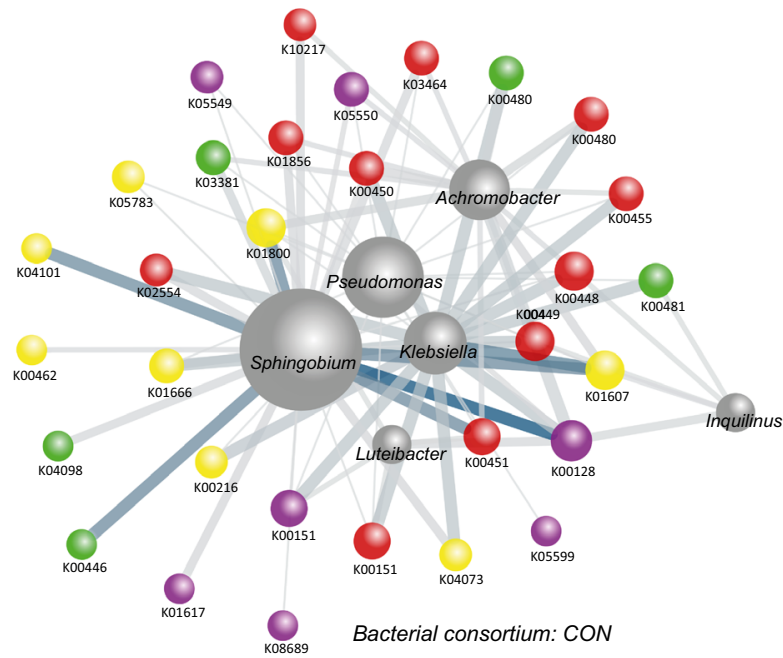

Fig. 4 Network visualization of the occurrence of genes in bacterial phylotypes involved in phenanthrene metabolism predicted by PICRUSt. Biadjacency matrix for consortia CON and I-CON (a). Bipartite network for consortium CON (b) and I-CON (c) showing the relationships between bacterial phylotypes and $\mathrm{KO}$ terms (functional

ecological perspective, which will allow deepening in general principles, microbiological theories and practical applications of the inoculation phenomenon (Mallon et al. 2015).

Previous studies of the consortium CON showed that its degradation efficiency is limited (59\%) (Festa et al. 2013). In this work we attempted to design a microbial consortium with higher biodegradation efficiency by inoculating CON with Sphingobium sp. 20006FA strain, that own several of the desirable traits for PAH degradation (Coppotelli et al. 2010) and was designated as a suitable candidate for bioaugmentation processes (Coppotelli et al. 2008).

The introduction of microorganisms into an environment is considered successful when the introduced species have the capacity to alter the interactions within the community, translated into changes in community structure (Mallon et al. 2015). It has been postulated that resource availability is the main factor that modulates biodiversity-invasion relationships by altering competitive interactions (Yang et al. 2017). The inoculated consortium I-CON was more efficient in degrading phenanthrene (78\%) than CON (59\%) and did not accumulate the toxic intermediate metabolite HNA (Fig. 1). The effective
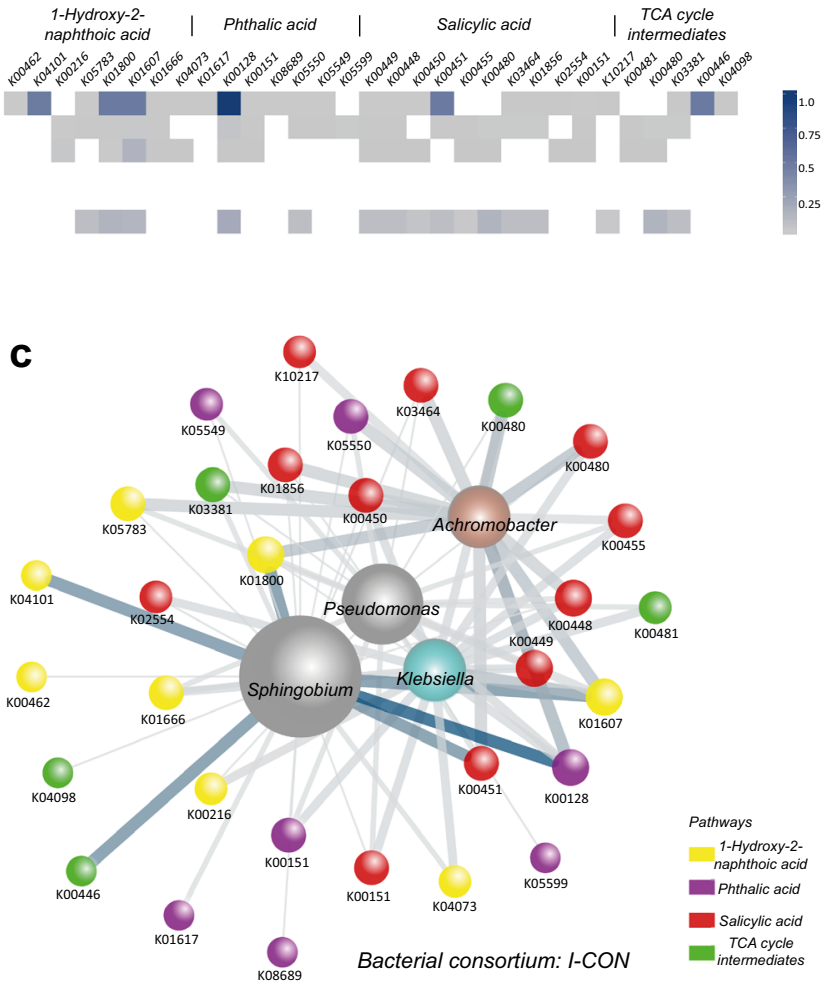

genes predicted by PICRUSt). Enzymes of phenanthrene degradation pathway are indicated in colors as follows: yellow dots, steps from phenanthrene to HNA; violet dots, steps from HNA to phthalic acid (PA); red dots, steps from HNA to salicylic acid, and green dots, steps from SA and PA to TCA intermediaries

communication among bacterial members of consortia enables the division of labor to perform complex tasks (Brenner et al. 2008). Higher efficiencies in biodegradation of crude oil were also observed when an indigenous bacterial consortium was inoculated with exogenous Bacillus subtillis (Tao et al. 2017).

Microorganisms in a new environment need to adapt to the environmental change and deal with novel interactions (Gómez et al. 2010). Here, changes in the degradation behavior in the natural consortium were caused by the presence of 20006FA strain, which could interact with some microorganisms within CON. Inoculation not only increased phenanthrene degradation efficiency of CON but also expanded its degradation potential (Table 1), incorporating the degrading capacities of the inoculated strain. However, Sphingobium sp. 20006FA strain, under the same conditions, showed higher phenanthrene degradation than I-CON. In the mutualistic relationships, the cross-fed nutrients can be either waste products valued by only one partner or nutrients valued by both partners (Mccully et al. 2017). In the last case, the competitive advantage of the recipient is in turn necessary to limit 


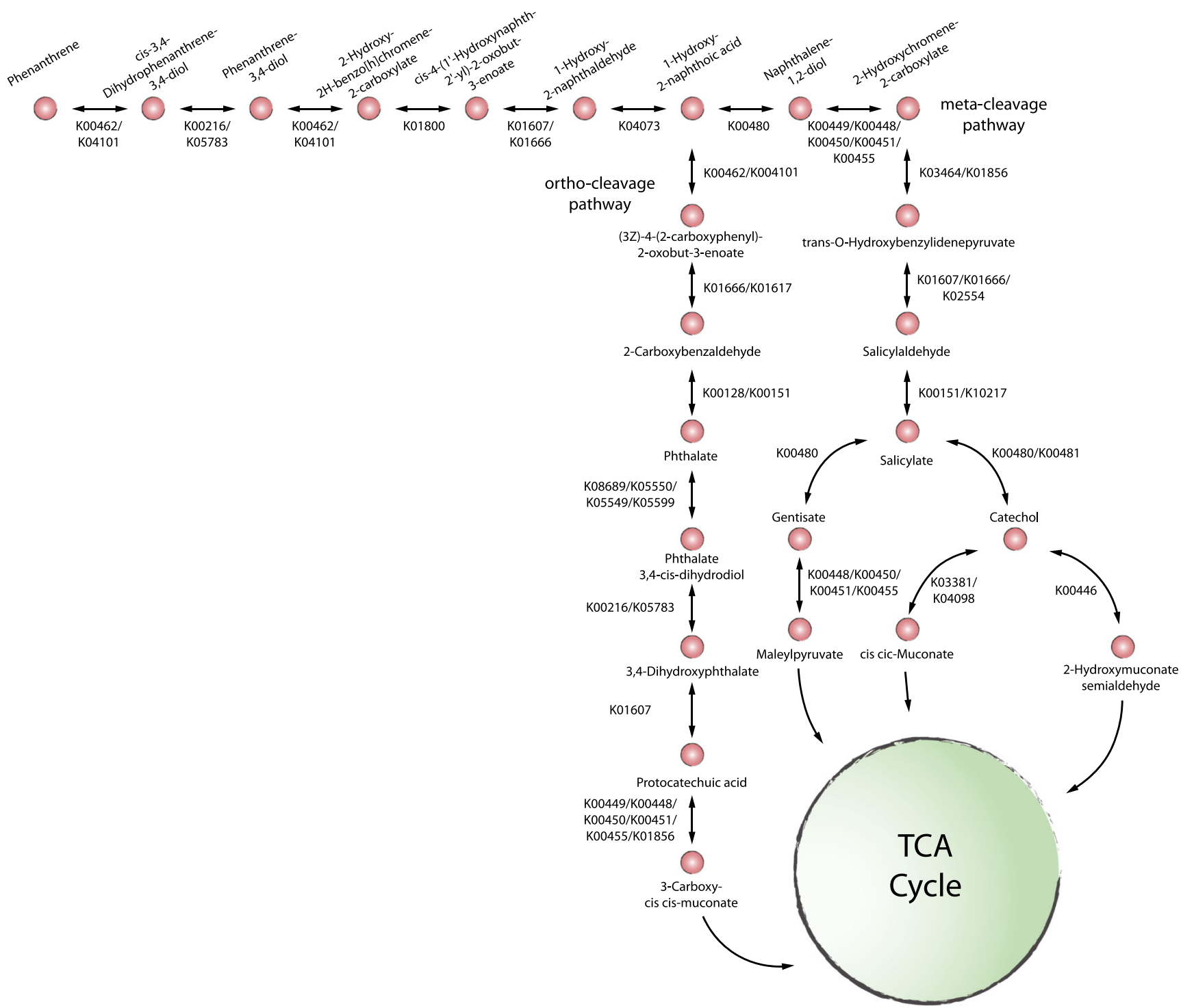

Fig. 5 Phenanthrene-degrading metabolic pathway using as reference the database of the Kyoto Encyclopedia of Genes and Genomes (KEGG) and the enzyme activities predicted by PICRUSt

reacquisition of the excreted nutrient by the producer, in our system, native Sphingobium sp. (Fig. 3 and Festa et al. (2013)) or the 20006FA strain, thereby to drive directionality nutrient exchange and to promote mutualism stability.

One of the possible cross-fed nutrients, the HNA, was accumulated by CON, but not by I-CON (Fig. 1). Changes in nutrient availability can switch between a large number of different interaction types, including mutualism, competition, and competitive exclusion (Hoek et al. 2016); in concordance with the PICRUSt prediction, the limitation in HNA degradation would not be related to lack of genetic capabilities, as shown in Fig. S1, but to cross-fed interaction established within $\mathrm{CON}$.

During phenanthrene degradation, 20006FA strain caused changes in the dynamics, composition, diversity, and proteomic profiles of CON (Figs. 2, 3, 4, and S2), which could be considered as an indicator of the inoculant establishment. DGGE (Fig. 2a) and proteomic (Fig. 2b) profiles supplied the clearest evidence of the inoculated strain establishment in I-CON by the observed predominant bands (yellow arrow) corresponding to the inoculant throughout the incubation period. Moreover, the difference in yellow colony number (with the same morphology as the inoculated strain, Fig. S3) between the CON and ICON over the incubation time (Fig. S2) would reveal a successful invasion, where the invader becomes an indigenous resident. A decrease in genetic diversity during phenanthrene degradation was observed in I-CON (Fig. 3 ), reflected by Shannon index $\left({ }^{1} \mathrm{D}\right)$ value (Table 2 ), indicating a displacement of other species by 20006FA. Resource competition is the main factor that affects the 
survival of newly introduced species in a bacterial community (Yang et al. 2017). Therefore, the successful invasion could indicate the participation of Sphingobium sp. 20006FA strain in the central carbon and energy flow, improving phenanthrene degradation.

In both consortia, the higher velocity of phenanthrene degradation (day 4) was concordant with the predominance of yellow colonies, characteristic of Sphingobium genus (Takeuchi et al. 2001). Furthermore, 16S rRNA gene pyrosequencing results revealed that the majority sequences present in CON and I-CON corresponded to Sphingobium genus (Fig. 3), known to be catabolically versatile and genetically adapted to different environmental conditions (Madueño et al. 2011; Waigi et al. 2015). New generation sequencing technologies provide information about the structure and diversity of a community revealing also the presence of low abundance members (Bohorquez et al. 2012). The analyses of the bacterial consortia composition revealed in I-CON a bacterial succession after inoculation. The sequences of Achromobacter sp. increased whereas Klebsiella sp. sequences decreased, and Inquilinus sp. and Luteibacter sp. were not detected, leading to a reduction in functional redundancy in I-CON (Fig. 3).

Although pyrosequencing analyses was not performed at day 15 , when phenanthrene degradation ceased in consortium $\mathrm{CON}$, changes observed in the heterotrophic cultivable bacteria and PAH-degrading bacteria counts (Fig. S2) and in DGGE profiles (Fig. 2a) would indicate a different bacterial succession in CON in comparison with I-CON. In CON, the diversity increased at expense of a decrease in the abundance of Sphingobium genus (Table 2 and Fig. 3).

Phenanthrene degradation improvement in consortium ICON could be explained not only by the inoculation with the 20006FA strain, but also by the increase in the relative abundance of Achromobacter genus (including Burkholderiales order), who despite not been predicted to own the initial dioxygenase, has several dioxygenases and enzymes that act in the low way of the phenanthrene pathway (Fig. 5). In a functional metagenomics analysis performed on $\mathrm{CON}$, a genome fragment belonging to Burkholderiales order was found, which encodes proteins related to one of the lower pathways (meta-cleavage of catechol) involved in PAH degradation (Festa et al. 2017). Sphingomonas and Achromobacter genera are the two major populations that could occupy the same ecological niche and have antagonistic relationships. Antagonistic effects were not observed in ICON cultures, where an increase in degradation efficiency and potential was observed (Fig. 1 and Table 1), involving a synergistic interaction. In relation to Klebsiella sp., some studies suggest that strains belonging to this genus have bioemulsifying activity in liquid culture with hydrocarbons (Toledo et al. 2008; Hua et al. 2010), that would reveal another function during biodegradation.
Despite competition is a fundamental driving force in evolution, it is paradoxical that cooperation exists at all. In cooperative systems, competition is best exemplified by the presence of individuals that do not cooperate (Dressler et al. 2017). These "cheaters" are often endowed with a fitness advantage over cooperators as they benefit from cooperation but do not share in the costs (Dressler et al. 2017), and should destroy cooperation. If during adaptation to the nutrient-limited cooperative environment (in our cultures the low solubility of phenanthrene determines the nutrient-limited), the maximum fitness gain of co-operators exceeds that of cheaters by at least the cost of cooperation, cooperators will win the race, and eventually purge the inferior cheaters (Waite and Shou 2012). It could be argued that in I-CON, the inoculation improved the cooperation between Sphingobium and Achromobacter genera, thereby, some competition could have been generated, and as a consequence, species in minor proportion (cheaters), Inquilinus sp. and Luteibacter sp., were not detected after inoculation.

Commonly, abundant microorganisms dominate the functionality of ecosystems and rare species that are retained by natural selection can become the most active population if they have a higher metabolic activity as soon as environmental conditions (nutrient status) change (De Roy et al. 2014). Functional redundancy is important to maintain the ecosystem active after perturbations (Mendes et al. 2015). A cooperative metabolic network was constructed by merging 16S rRNA gene sequence analyses of our consortium, obtained from a chronically contaminated soil $(\mathrm{CON})$. Bacterial community diversity, composition, and prediction of functional capacities (PICRUSt) of bacterial consortia members were obtained (Figs. 4 and 5). In this metabolic network, different microbial groups contributed to each step in phenanthrene degradation. It was predicted that Sphingobium genus played a key role in the initial degradation reactions. Previous reports indicated that Sphingomonads are able to adapt and become abundant when PAH are delivered in the environment (Stolz 2009; Khara et al. 2014) and many members of Pseudomonas (Balashova et al. 2001) are capable of degrading various PAHs. However, in our consortia, the unique member predicted to be capable of initiating phenanthrene degradation was Sphingobium sp. Other populations present in both consortia could remain in the system due to their ability to assimilate diverse metabolites from other microbes.

The analyses of functional genes demonstrated that populations present in the bacterial consortium $\mathrm{CON}$ could be involved into different steps of phenanthrene degradation, genes encoding for 21 enzymes involved in the transformation reactions from HNA up to the TCA cycle were predicted. The constructed network using bacterial phylotypes connected enzymes involved in degradation and indicated functional 
redundancy in all the steps of the pathway, with exception of steps 1 and 10, which enzymes were only encoded in Sphingobium sp. (Figs.4 and 5).

PAH degradation in the environment is performed by cooperative microbes, although some axenic microbial cultures are themselves able to completely mineralize PAHs (Zhao et al. 2016). Members of Sphingobium genus are involved in all the steps needed for metabolizing phenanthrene to TCA intermediaries. While Sphingobium genus could perform the biodegradation process individually (Fig. 4), which were supported by metabolite analyses based on cultures (Festa et al. 2013), in consortium CON, this bacterial taxa is grouped together with other bacteria with or without degrading capacities. This could indicate that interactions formed by coadaptation/evolution were still necessary for these bacteria and could optimize its functionality (Stadie et al. 2013). Interactions formed by coadaptation/evolution in a special community occurred not only between "functional bacteria" with degrading ability (showed direct effect on hydrocarbons) but also between "functional bacteria" and bacteria without degrading ability, that could promote/inhibit the degrading process (Wang et al. 2016).

\section{Conclusion}

The present work provides the first insides of a natural phenanthrene-degrading consortium that has been characterized by new generation sequencing technologies, inoculated with an exogenous strain in order to study the interaction between the inoculant and the community during PAH degradation.

Sphingobium sp. 20006FA strain accomplished some of the key features of invasions, such as rapid adaptation and permanence over time, which should be necessary characteristics for microorganism to establish and diffuse successfully according to the ecological interactions between species. This was reflected by the increase in degradation efficiency achieved by I-CON, the prevention of the accumulation of HNA, and the changes produced on the bacterial structure of the natural consortium CON.

Our prediction showed that specific bacterial taxa dominate in different steps of the degrading process. In I-CON, Sphingobium sp. was predicted as the unique functional bacterial group responsible for the initial attack to phenanthrene; the significant increase in Achromobacter sp., could indicated that this group contribute most to the second step and to the degradation of HNA to phthalic acid or to salicylic acid. Other consortia members, Pseudomonas sp. and Klebsiella sp., were predicted to consume intermediate metabolites.

Despite the inoculation improving the cooperation between Sphingobium and Achromobacter genera, some competition could have occurred, and therefore, species in minor proportion (cheaters), Inquilinus sp. and Luteibacter sp., were not detected after inoculation. These changes could indicate that the introduced strain is highly competitive and did adapt to the prevailing conditions within CON during phenanthrene degradation.

Funding This research was partially supported by the Agencia Nacional de Promoción Científica y Tecnológica (PICT 2013-0103). Festa S. and Macchi M. have doctoral and postdoctoral fellowships supported by CONICET. Morelli I.S. is a research member of CIC-PBA. Coppotelli B.M. is a research member of CONICET.

\section{References}

Balashova NV, Stolz A, Knackmuss HJ, Kosheleva IA, Naumov AV, Boronin AM (2001) Purification and characterization of a salicylate hydroxylase involved in 1-hydroxy-2-naphthoic acid hydroxylation from the naphthalene and phenanthrene-degrading bacterial strain Pseudomonas putida BS202-P1. Biodegradation 12:179-188. https://doi.org/10.1023/A:1013126723719

Bohorquez LC, Delgado-Serrano L, López G, Osorio-Forero C, KlepacCeraj V, Kolter R, Junca H, Baena S, Zambrano MM (2012) Indepth characterization via complementing culture-independent approaches of the microbial Community in an Acidic hot Spring of the Colombian Andes Laura. Microb Ecol 63:103-115. https://doi.org/ 10.1007/s00248-011-9943-3ENVIRONMENTAL

Brenner K, You L, Arnold FH (2008) Engineering microbial consortia: a new frontier in synthetic biology. Trends Biotechnol 26:483-489. https://doi.org/10.1016/j.tibtech.2008.05.004

Caporaso JG, Kuczynski J, Stombaugh J et al (2010) QIIME allows analysis of high- throughput community sequencing data intensity normalization improves color calling in SOLiD sequencing. Nat Publ Group 7:335-336. https://doi.org/10.1038/nmeth0510-335

Cerniglia CE (1993) Biodegradation of polycyclic aromatic hydrocarbons. 4:331-338. https://doi.org/10.1016/0958-1669(93)90104-5

Cerniglia CE, Yang SK (1984) Stereoselective metabolism of anthracene and phenanthrene by the fungus Cunninghamella elegans. Appl Environ Microbiol 47:119-124

Chiu CH, Chao A (2014) Distance-based functional diversity measures and their decomposition: a framework based on hill numbers. PLoS One 9:e100014. https://doi.org/10.1371/journal.pone.0100014

Comeau AM, Douglas GM, Langille MGI (2017) Microbiome helper: a custom and streamlined workflow for microbiome research. mSystems 2:e00127-e00116. https://doi.org/10.1128/mSystems. 00127-16

Coppotelli BM, Ibarrolaza A, Del Panno MT, Morelli IS (2008) Effects of the inoculant strain Sphingomonas paucimobilis 20006FA on soil bacterial community and biodegradation in phenanthrenecontaminated soil. Microb Ecol 55:173-183. https://doi.org/10. 1007/s00248-007-9265-7

Coppotelli BM, Ibarrolaza A, Dias RL, del Panno MT, Berthe-Corti L, Morelli IS (2010) Study of the degradation activity and the strategies to promote the bioavailability of phenanthrene by Sphingomonas paucimobilis strain 20006FA. Microb Ecol 59:266-276. https:// doi.org/10.1007/s00248-009-9563-3

De Lorenzo V, Pieper D, Ramos JL (2013) From the test tube to the environment-and back. Environ Microbiol 15:6-11. https://doi.org/ 10.1111/j.1462-2920.2012.02896.x 
De Roy K, Marzorati M, Van den Abbeele P et al (2014) Synthetic microbial ecosystems: an exciting tool to understand and apply microbial communities. Environ Microbiol 16:1472-1481. https://doi. org/10.1111/1462-2920.12343

Desai C, Pathak H, Madamwar D (2010) Advances in molecular and "omics" technologies to gauge microbial communities and bioremediation at xenobiotic/anthropogen contaminated sites. Bioresour Technol 101:1558-1569. https://doi.org/10.1016/j.biortech.2009. 10.080

Dressler MD, Clark CJ, Thachettu CA, Zakaria Y, Eldakar OT, Smith RP (2017) Synthetically engineered microbes reveal interesting principles of cooperation. Front Chem Sci Eng 11:3-14. https://doi.org/ 10.1007/s11705-016-1605-z

Edgar RC, Haas BJ, Clemente JC, Quince C, Knight R (2011) UCHIME improves sensitivity and speed of chimera detection. Bioinformatics 27:2194-2200. https://doi.org/10.1093/bioinformatics/btr381

Festa S, Coppotelli BM, Morelli IS (2013) Bacterial diversity and functional interactions between bacterial strains from a phenanthrenedegrading consortium obtained from a chronically contaminatedsoil. Int Biodeterior Biodegrad 85:42-51. https://doi.org/10.1016/j. ibiod.2013.06.006

Festa S, Coppotelli BM, Morelli IS (2016) Comparative bioaugmentation with a consortium and a single strain in a phenanthrenecontaminated soil: impact on the bacterial community and biodegradation. Appl Soil Ecol 98:8-19. https://doi.org/10.1016/j.apsoil. 2015.08.025

Festa S, Coppotelli BM, Madueño L, Loviso CL, Macchi M, Neme Tauil RM, Valacco MP, Morelli IS (2017) Assigning ecological roles to the populations belonging to a phenanthrene-degrading bacterial consortium using omic approaches. PLoS One 12:1-21. https:// doi.org/10.1371/journal.pone.0184505

Friedman J, Gore J (2017) Ecological systems biology: The dynamics of interacting populations. Curr Opin Syst Biol 1:114-121. https://doi. org/10.1016/j.coisb.2016.12.001

Gómez JP, Bravo GA, Brumfield RT, Tello JG, Cadena CD (2010) A phylogenetic approach to disentangling the role of competition and habitat filtering in community assembly of Neotropical forest birds. J Anim Ecol 79:1181-1192. https://doi.org/10.1111/j.1365-2656. 2010.01725.x

Gotelli NJ, Chao A (2013) Measuring and estimating species richness, species diversity, and biotic similarity from sampling data. Elsevier Ltd

Hill MO (1973) Diversity and evenness: a unifying notation and its consequences. Ecology 54:427-432. https://doi.org/10.2307/1934352

Hoek TA, Axelrod K, Biancalani T, Yurtsev EA, Liu J, Gore J (2016) Resource availability modulates the cooperative and competitive nature of a microbial cross-feeding mutualism. PLoS Biol 14:117. https://doi.org/10.1371/journal.pbio. 1002540

Hua X, Wu Z, Zhang H, Lu D, Wang M, Liu Y, Liu Z (2010) Degradation of hexadecane by Enterobacter cloacae strain TU that secretes an exopolysaccharide as a bioemulsifier. Chemosphere 80:951-956. https://doi.org/10.1016/j.chemosphere.2010.05.002

Kazunga C, Aitken MD (2000) Products from the incomplete metabolism of pyrene by polycyclic aromatic hydrocarbon-degrading bacteria. Appl Environ Microbiol 66:1917-1922. https://doi.org/10.1128/ AEM.66.5.1917-1922.2000

Khara P, Roy M, Chakraborty J, Ghosal D, Dutta TK (2014) Functional characterization of diverse ring-hydroxylating oxygenases and induction of complex aromatic catabolic gene clusters in Sphingobium sp. PNB. FEBS Open Bio 4:290-300. https://doi. org/10.1016/j.fob.2014.03.001

Kopylova E, Noé L, Touzet H (2012) SortMeRNA: fast and accurate filtering of ribosomal RNAs in metatranscriptomic data. Bioinformatics 28:3211-3217. https://doi.org/10.1093/ bioinformatics/bts611
Langille MGI, Zaneveld J, Caporaso JG, McDonald D, Knights D, Reyes JA, Clemente JC, Burkepile DE, Vega Thurber RL, Knight R, Beiko RG, Huttenhower C (2013) Predictive functional profiling of microbial communities using 16S rRNA marker gene sequences. Nat Biotechnol 31:814-821. https://doi.org/10.1038/nbt.2676

Macchi M, Martinez M, Tauil RMN, Valacco MP, Morelli IS, Coppotelli BM (2018) Insights into the genome and proteome of Sphingomonas paucimobilis strain 20006FA involved in the regulation of polycyclic aromatic hydrocarbon degradation. World J Microbiol Biotechnol 34:7. https://doi.org/10.1007/s11274-0172391-6

Madueño L, Coppotelli BM, Alvarez HM, Morelli IS (2011) Isolation and characterization of indigenous soil bacteria for bioaugmentation of PAH contaminated soil of semiarid Patagonia, Argentina. Int Biodeterior Biodegrad 65:345-351. https://doi.org/10.1016/j.ibiod. 2010.12.008

Mallon CA, Van Elsas JD, Salles JF (2015) Microbial invasions: the process, patterns, and mechanisms. Trends Microbiol 23:719-729. https://doi.org/10.1016/j.tim.2015.07.013

Mccully AL, Lasarre B, Mckinlay JB (2017) Recipient-biased competition for an intracellularly generated cross-fed nutrient is required for coexistence of microbial mutualists. MBio 8:1-13. https://doi.org/ 10.1128/mBio.01620-17

Megharaj M, Ramakrishnan B, Venkateswarlu K, Sethunathan N, Naidu R (2011) Bioremediation approaches for organic pollutants: a critical perspective. Environ Int 37:1362-1375. https://doi.org/10.1016/ j.envint.2011.06.003

Mendes LW, Tsai SM, Navarrete AA, de Hollander M, van Veen JA, Kuramae EE (2015) Soil-borne microbiome: linking diversity to function. Microb Ecol 70:255-265. https://doi.org/10.1007/ s00248-014-0559-2

Mercier C, Boyer F, Bonin A, Coissac E (2013) SUMATRA and SUMACLUST: fast and exact comparison and clustering of sequences. Available: https://git.metabarcoding.org/obitools/ sumaclust

Muyzer G, Smalla K (1998) Application of denaturing gradient gel electrophoresis (DGGE) and temperature gradient gel electrophoresis (TGGE) in microbial ecology. Antonie Van Leewenhoek 73:127141. https://doi.org/10.1023/A:1000669317571

Muyzer G, de Waal EC, Uitterlinden AG (1993) Profiling of complex microbial populations by denaturing gradient gel electrophoresis analysis of polymerase chain reaction-amplified genes coding for 16S rRNA. Appl Environ Microbiol 59:695-700

Pavlopoulos GA, Secrier M, Moschopoulos CN, Soldatos TG, Kossida S, Aerts J, Schneider R, Bagos PG (2011) Using graph theory to analyze biological networks. BioData Min 4:1-27. https://doi.org/10. 1186/1756-0381-4-10

Pavlopoulos GA, Kontou PI, Pavlopoulou A et al (2018) Bipartite graphs in systems biology and medicine: a survey of methods and applications. GigaScience 7(4):giy014. https://doi.org/10.1093/ gigascience/giy014

Quast C, Pruesse E, Yilmaz P, et al (2012) The SILVA ribosomal RNA gene database project: Improved data processing and web-based tools. Nucleic Acids Res 41:590-596. https://doi.org/10.1093/nar/ gks1219

Reasoner DJ, Geldreich EE (1985) A new medium for the enumeration and subculture of bacteria from potable water. Appl Environ Microbiol 49:1-7. https://doi.org/10.1016/s0016-7037(99)00322-1

Rognes T, Flouri T, Nichols B, Quince C, Mahé F (2016) VSEARCH: a versatile open source tool for metagenomics. PeerJ 4:e2584. https:// doi.org/10.7717/peerj.2584

Rungrassamee W, Klanchui A, Chaiyapechara S, Maibunkaew S, Tangphatsornruang S, Jiravanichpaisal P, Karoonuthaisiri N (2013) Bacterial population in intestines of the black tiger shrimp (Penaeus monodon) under different growth stages. PLoS One 8:e60802. https://doi.org/10.1371/journal.pone.0060802 
Shannon P, Markiel A, Ozier O, Baliga NS, Wang JT, Ramage D, Amin N, Schwikowski B, Ideker T (2003) Cytoscape: a software environment for integrated models of biomolecular interaction networks. Genome Res 13:2498-2504. https://doi.org/10.1101/gr.1239303

Stadie J, Gulitz A, Ehrmann MA, Vogel RF (2013) Metabolic activity and symbiotic interactions of lactic acid bacteria and yeasts isolated from water kefir. Food Microbiol 35:92-98. https://doi.org/10.1016/j.fm. 2013.03.009

Stolz A (2009) Molecular characteristics of xenobiotic-degrading sphingomonads. Appl Microbiol Biotechnol 81:793-811. https:// doi.org/10.1007/s00253-008-1752-3

Su Z, Dai T, Tang Y, Tao Y, Huang B, Mu Q, Wen D (2018) Sediment bacterial community structures and their predicted functions implied the impacts from natural processes and anthropogenic activities in coastal area. Mar Pollut Bull 131:481-495. https://doi.org/10.1016/ j.marpolbul.2018.04.052

Takeuchi M, Hamana K, Hiraishi A (2001) Proposal of the genus Sphingomonas sensu stricto and three new genera. Int J Syst Evol Microbiol 51:1405-1417. https://doi.org/10.1099/00207713-51-41405

Tamaki H, Wright CL, Li X, Lin Q, Hwang C, Wang S, Thimmapuram J, Kamagata Y, Liu WT (2011) Analysis of 16S rRNA amplicon sequencing options on the roche/454 next-generation titanium sequencing platform. PLoS One 6:6-8. https://doi.org/10.1371/ journal.pone. 0025263

Tao K, Liu X, Chen X, Hu X, Cao L, Yuan X (2017) Biodegradation of crude oil by a defined co-culture of indigenous bacterial consortium and exogenous Bacillus subtilis. Bioresour Technol 224:327-332. https://doi.org/10.1016/j.biortech.2016.10.073

Timmis KN, Pieper DH (1999) Bacteria designed for bioremediation. Trends Biotechnol 17:201-204. https://doi.org/10.1016/S01677799(98)01295-5

Toledo FL, Gonzalez-Lopez J, Calvo C (2008) Production of bioemulsifier by Bacillus subtilis, Alcaligenes faecalis and Enterobacter species in liquid culture. Bioresour Technol 99:8470 8475. https://doi.org/10.1016/j.biortech.2007.08.055

Vecchioli GI, Del Panno MT, Painceira MT (1990) Use of selected autochthonous soil bacteria to enhanced degradation of hydrocarbons in soil. Environ Pollut 67:249-258. https://doi.org/10.1016/02697491(90)90190-N

Větrovský T, Baldrian P (2013) The variability of the 16S rRNA gene in bacterial genomes and its consequences for bacterial community analyses. PLoS One 8:1-10. https://doi.org/10.1371/journal.pone. 0057923

Waigi MG, Kang F, Goikavi C, Ling W, Gao Y (2015) Phenanthrene biodegradation by sphingomonads and its application in the contaminated soils and sediments: a review. Int Biodeterior Biodegrad 104: 333-349. https://doi.org/10.1016/j.ibiod.2015.06.008

Waite AJ, Shou W (2012) Adaptation to a new environment allows cooperators to purge cheaters stochastically. Proc Natl Acad Sci U S A 109:19079-19086. https://doi.org/10.1073/pnas.1210190109
Wanapaisan P, Laothamteep N, Vejarano F, Chakraborty J, Shintani M, Muangchinda C, Morita T, Suzuki-Minakuchi C, Inoue K, Nojiri H, Pinyakong O (2018) Synergistic degradation of pyrene by five culturable bacteria in a mangrove sediment-derived bacterial consortium. J Hazard Mater 342:561-570. https://doi.org/10.1016/j. jhazmat.2017.08.062

Wang H, Wang B, Dong W, Hu X (2016) Co-acclimation of bacterial communities under stresses of hydrocarbons with different structures. Sci Rep 6:1-12. https://doi.org/10.1038/srep34588

Wickham H (2016) ggplot2 - elegant graphics for data analysis. SpringerVerlag, New York 77. https://doi.org/10.1007/978-3-319-24277-4

Wrenn BA, Venosa AD (1996) Selective enumeration of aromatic and aliphatic hydrocarbon degrading bacteria by a most-probablenumber procedure. Can J Microbiol 42:252-258. https://doi.org/ 10.1139/m96-037

Yang T, Wei Z, Friman VP, Xu Y, Shen Q, Kowalchuk GA, Jousset A (2017) Resource availability modulates biodiversity-invasion relationships by altering competitive interactions. Environ Microbiol 19:2984-2991. https://doi.org/10.1111/1462-2920.13708

Yuan SY, Wei SH, Chang BV (2000) Biodegradation of polycyclic aromatic hydrocarbons by a mixed culture. Chemosphere 41:14631468

Zafra G, Absalón ÁE, Anducho-Reyes MÁ, Fernandez FJ, CortésEspinosa DV (2017) Construction of PAH-degrading mixed microbial consortia by induced selection in soil. Chemosphere 172:120 126. https://doi.org/10.1016/j.chemosphere.2016.12.038

Zhang J, Kobert K, Flouri T, Stamatakis A (2014) PEAR: a fast and accurate Illumina paired-end reAd mergeR. Bioinformatics 30: 614-620. https://doi.org/10.1093/bioinformatics/btt593

Zhao HP, Wang L, Ren JR, Li Z, Li M, Gao HW (2008) Isolation and characterization of phenanthrene-degrading strains Sphingomonas sp. ZP1 and Tistrella sp. ZP5. J Hazard Mater 152:1293-1300. https://doi.org/10.1016/j.jhazmat.2007.08.008

Zhao JK, Li XM, Ai GM, Deng Y, Liu SJ, Jiang CY (2016) Reconstruction of metabolic networks in a fluoranthene-degrading enrichments from polycyclic aromatic hydrocarbon polluted soil. J Hazard Mater 318:90-98. https://doi.org/10.1016/j.jhazmat.2016. 06.055

Zhao Q, Yue S, Bilal M, et al (2017) Comparative genomic analysis of 26 Sphingomonas and Sphingobium strains: Dissemination of bioremediation capabilities, biodegradation potential and horizontal gene transfer. Sci Total Environ 609:1238-1247. https://doi.org/10.1016/ j.scitotenv.2017.07.249

Publisher's note Springer Nature remains neutral with regard to jurisdictional claims in published maps and institutional affiliations. 